\title{
ON THE TIME AND CELL DEPENDENCE OF THE COARSE-GRAINED ENTROPY. I
}

\author{
P. HOYNINGEN-HUENE \\ Institut für Theoretische Physik der Universität Zürich, \\ CH-8001 Zurich, Switzerland
}

Received 4 July 1975

\begin{abstract}
We consider a finite, thermally isolated, classical system which passes from an equilibrium state $\mathscr{A}$ by the removal of an internal constraint to another equilibrium state $\mathscr{B}$ after an empirical relaxation time. In the phase space of the system, cells are introduced according to the set of measuring instruments used and their experimental inaccuracies. It is shown that the coarsegrained entropy $S_{\mathrm{cg}}(t)$ tends to its new equilibrium value in general faster than the expectation values of the macroscopic variables to their new equilibrium values. We then investigate the dependence of $S_{\mathrm{cg}}(t)$ on the size of the phase cells. For fixed $t$, we find a lower bound on $S_{\mathrm{cg}}(t)$ by going to the limit of infinite accuracy of the measuring instruments. In the limit $t \rightarrow \infty$, this lower bound on $S_{\mathrm{cg}}(t)$ also converges to the equilibrium entropy of $\mathscr{B}$. These properties strongly support the opinion that $S_{\mathrm{cg}}(t)$ is a proper microscopic expression for the entropy for equilibrium and nonequilibrium. Finally, explicit calculations of $S_{\mathrm{cg}}(t)$ for the model of a point particle enclosed in a one-dimensional box are presented which confirm the general results.
\end{abstract}

\section{Introduction}

The problem of defining a microscopic expression for the entropy has had a history of more than 100 years. It is still a challenging problem, and at the moment, there is no expression which is both sufficiently general and commonly accepted by the scientific community. This situation is felt to be uncomfortable since a proper microscopic expression for the entropy is the key to an accurate understanding of the Second Law.

The first microscopic expression for the entropy is due to L. Boltzmann, but his expression is only valid under cerlain restrictive conditions on the system considered, for instance that the density is fairly low. The Boltzmann entropy generalizes the concept of the thermostatic entropy into the nonequilibrium domain. To get rid of the restrictions on the properties of the system, however, seems to be a yet unsolved problem. 
J.W. Gibbs in his statistical mechanical approach found an expression for the entropy perfectly valid for all kinds of systems in equilibrium. But the Gibbs entropy failed to show the characteristic feature of the entropy given by the Second Law that is roughly speaking its. nondecrease for adiabatically isolated systems, since the Gibbs entropy is a constant of the motion. Thus, the Gibbs entropy is insufficient in the opposite sense to the Boltzmann entropy: valid for all kinds of systems indeed, but only for equilibrium situations.

The historically first attempt to escape this difficulty was to introduce the wellknown coarse-graining which altered the definition of the Gibbs entropy in such a way that its equilibrium properties were kept, but the constancy in time was destroyed. The second attempt which shall not be dealt with in this paper was to give up the idea of a strictly isolated system and to modify the Liouville equation in such a way that the original Gibbs entropy changes in time.

The discussion about coarse-graining is still in process. In this paper, we shall only deal with the question whether coarse-graining may lead to an appropriate microscopic expression for the entropy for both equilibrium and nonequilibrium. To this aim, we start in section 2 with some preliminaries fixing the physical situation for which we define entropy and restating the postulates on a microscopic expression for the entropy. In section 3 , we discuss the problem of the unique choice of a set of phase cells for a given physical situation. Section 4 dcals with the properties of the coarse-grained entropy. A model calculation confirming the general results is given in section 5 . The paper closes in section 6 with a discussion and summary.

\section{Preliminaries}

We consider a finite thermally isolated classical system which shall be observed macroscopically by means of $r$ quantities $A_{1}^{\text {exp }}, \ldots, A_{r}^{\text {exp }}$, where the superscript exp stands for experimental. Microscopically, the system is described in a subset $\Sigma$ of its phase space $\Gamma: \Sigma$ may be an energy surface $H(x)=E$ or an energy shell $E \leqslant H(x) \leqslant E+\Delta E$, where $H(x)$ is the hamiltonian and $x \in \Gamma$. The corresponding invariant measure in $\Sigma$ is $\mathrm{d} \mu=\mathrm{d} \sigma /|\operatorname{grad} H|$ where $\mathrm{d} \sigma$ is the surface element, for the energy surface, or $\mathrm{d} \mu=\mathrm{d} x$, where $\mathrm{d} x$ is the Lebesgue measure, for the energy shell, respectively. $\Sigma$ is assumed to be finite, i.e. $\mu(\Sigma)<\infty$.

In the equilibrium states of the system, the phenomenological entropy $S$ is defined as usually: if $\mathscr{A}=\left(A_{1}^{\text {exp }}, \ldots, A_{r}^{\text {exp }}\right)$ and $\mathscr{B}=\left(\bar{A}_{1}^{\exp }, \ldots, A_{r}^{\text {exp }}\right)$ are equilibrium states and $S(\mathscr{A})$ has been chosen as some constant, then

$$
S(\mathscr{B})=S(\mathscr{A})+\int_{\mathscr{A}}^{\mathscr{B}} \delta Q_{\mathrm{rev}} / T .
$$


Experimentally, we know the following properties of $S$ :

(E1) $S$ is a single-valued function.

(E2) $S$ is additive for independent systems (provided $S(\mathscr{A})$ has been chosen properly).

(E3) The Second Law for thermally isolated systems: if the removal of an internal constraint forces the system to pass from equilibrium state $\mathscr{A}$ to equilibrium state $\mathscr{B}$, then $S(\mathscr{B}) \geqslant S(\mathscr{A})^{\ddagger}$.

For the definition of a microscopic expression for $S$ for equilibrium and nonequilibrium, we restrict ourselves to the following model situation in which the system leaves an equilibrium state $\mathscr{A}$ by the removal of an internal constraint and reaches thereafter another equilibrium state $\mathscr{B}$.

$t<0$ : The system has an internal constraint and is in an equilibrium state $\mathscr{A}$.

Assumption 1: This state $\mathscr{A}$ is described in $\Sigma$ uniquely by an $N$-particle distribution function $\varrho_{0}(x)$, which is bounded. One may fulfil assumption 1 by postulating ergodic properties for the system. Supposing that the internal constraint makes only a subset $\Sigma^{\prime}$ of $\Sigma$ with $\mu\left(\Sigma^{\prime}\right)>0$ accessible to the system, and that the system is ergodic in $\Sigma^{\prime}$, then $\varrho_{0}(x)$ is determined uniquely to be identical with the microcanonical distribution function in $\Sigma^{\prime 34}$ ) and to be zero otherwise:

$$
\begin{aligned}
\varrho_{0}(x) & =\left(\int_{\Sigma^{\prime}} \mathrm{d} \mu\right)^{-1}, & & x \in \Sigma^{\prime} \\
& =0 & & x \in \Sigma \backslash \Sigma^{\prime} .
\end{aligned}
$$

$t=0$ : The internal constraint is being removed.

$t \geqslant 0$ : The evolution of the system is given by $g(x ; t)$ which is determined by $\varrho(x ; 0)=\varrho_{0}(x)$ and the Liouville equation characterizing the system; since $\varrho_{0}(x)$ is bounded, $\varrho(x ; t)$ is uniformly bounded for all times due to the Liouville theorem:

$$
\varrho(x ; t) \leqslant B \quad \text { for all } t .
$$

Assumption 2: $\varrho(x, t)$ is weakly convergent to the microcanonical distribution function in $\Sigma$

$$
\varrho_{\mathrm{mic}}(x):=\left(\int_{2} \mathrm{~d} \mu\right)^{-1}
$$

i.e.

$$
\lim _{t \rightarrow \infty} \int_{\Sigma} \varrho(x ; t) f(x) \mathrm{d} \mu=\int_{\Sigma} \varrho_{\text {mic }}(x) f(x) \mathrm{d} \mu \quad \text { for all } \quad f \in L_{2}(\Sigma, \mu) .
$$

Eq. (2.5) is fulfilled for example for a system having the ergodic property mixing ${ }^{35}$ ).

₹ We do not intend to enter the discussion how to formulate the Second Law in its most elegant and complete form; the reader is referred to the discussion in the literature, e.g. refs. 1-7. 
The macroscopic description of the approach to equilibrium involves the concept of the relaxation time $\tau_{\text {rel }}$. In our context, we mean by $\tau_{\text {rel }}$ the time after which the slowest relaxing macroscopic variable has reached its new equilibrium value within the experimental accuracy. In other words, a macroscopic observer states in the case of our model situation that the system is in its new equilibrium state $\mathscr{B}$ for times $t \geqslant \tau_{\text {rel }}$.

Now, how to define a microscopic expression $S_{\mathrm{mic}}$ for $S$, generalizing it to the nonequilibrium domain? We have to meet the following postulates:

(P1) The definition of $S_{\text {mic }}$ is universal in the sense that $S_{\text {mic }}$ is defined for all kinds of systems in both equilibrium and nonequilibrium states.

(P2) $S_{\text {mic }}$ depends essentially on microscopic quantities.

(P3) For the equilibrium states, $S_{\text {mic }}$ reproduces all thermodynamic properties of $S$.

(P4) If the system passes from equilibrium state $\mathscr{A}$ to equilibrium state $\mathscr{B}$ in $\tau_{\text {re1 }}$, and if $S_{\text {mic }}(t \leqslant 0)=S(\mathscr{A})$, then $S_{\text {mic }}(t)=S(\mathscr{B})$ within the experimental accuracy for $t \geqslant \tau_{\mathrm{re} 1}$.

Postulates (P1), (P2) and (P3) are evident. (P4) is the linking postulate between the equilibrium and nonequilibrium domain. It states nothing about the behavior of $S_{\mathrm{mic}}(t)$ for the time in which the system is macroscopically out of equilibrium. This is a consequence of the fact that the entropy is defined experimentally only for equilibrium states (e.g. ref. 8). In irreversible thermodynamics, however, a positive entropy production is often assumed, to which corresponds a postulate $\mathrm{d} S_{\text {mic }}(t) / \mathrm{d} t \geqslant 0$ or equivalently an $\mathrm{H}$-theorem. Many authors indeed postulate a monotonic increase for the entropy (e.g. refs. 9-15, 33). In the thermodynamic limit, in particular, this may be convenient and/or simple, but it is still an assumption which is not necessary in order to get agreement with experiments (e.g. refs. 16,17 ).

\section{Coarse-graining}

Coarse-graining has been introduced in the early days of statistical mechanics by Gibbs $^{18}$ ) and the Ehrenfest's ${ }^{19}$ ). The basic aim is to modify the Gibbs entropy

$$
S_{\mathrm{G}}:=-k \int_{\underline{L}} \varrho \ln \varrho \mathrm{d} \mu
$$

in such a way that the equilibrium properties of $S_{\mathrm{G}}$ are maintained, the constancy in time for a nonequilibrium situation, however, is abandoned. Clearly, this second goal can only be achieved by replacing $\varrho(x ; t)$ by another function which does not fulfil the Liouville equation. The intuitive idea to construct this function stems from the fact that during the time evolution $g(x ; t)$ spreads out in $\Sigma$ in thinner and thinner filaments without ever becoming uniform. A coarse observa- 
tion of $Q(x ; t)$, however, leads to a function $P(x ; t)$ which may indeed approach a uniform distribution in $\Sigma$, and does not fulfil the Liouville equation.

Mathematically, this "coarse look" at $\varrho(x ; t)$ is realized by the introduction of a finite set of "phase-cells" $\left\{\Omega_{j}\right\}$, i.e. a set of measurable subsets of $\Sigma$ with the properties

$$
\begin{aligned}
& \mu\left(\bigcup_{j} \Omega_{j}\right)=\mu(\Sigma), \\
& \mu\left(\Omega_{i} \cap \Omega_{j}\right)=0 \quad \text { for } i \neq j, \\
& \mu\left(\Omega_{j}\right)>0 .
\end{aligned}
$$

The "coarse-grained $N$-particle distribution function" $P(x ; t)$ is then obtained by averaging $\varrho(x ; t)$ over the cells, i.e.

$$
\left.P(x ; t)\right|_{x \in \Omega_{j}}:=\left(\mu\left(\Omega_{j}\right)\right)^{-1} \int_{\Omega_{j}} \varrho(x ; t) \mathrm{d} \mu=: P_{j}(t) .
$$

Before we proceed in defining the entropy functional, we have to discuss the following problems: which set of phase cells should be chosen for a particular experimental situation, and how can this choice be justified physically since up to this point, the introduction of phase cells has been an ad-hoc procedure.

Clearly the coarse structure of $\Sigma$ must have something to do with the coarse, that is macroscopic observation of the system. Within the context of the derivation of a master equation, van Kampen has introduced phase cells according to the measuring instruments used and their experimental accuracies ${ }^{20.21}$ ). The measurement of the macroscopic quantities $A_{i}^{\text {exp }}, i=1, \ldots, r$, contains inaccuracies $\delta_{i}, i=1, \ldots, r$, whose amounts may depend on the actual value $a_{i}$ of $A_{i}^{\text {exp }}$. Thus, the range of all values accessible to $A_{i}^{\text {exd }}$ may be divided into intervals of length $\delta_{i}$ :

$$
\ldots, a_{i}^{(-2)}, a_{i}^{(-1)}, a_{i}^{(0)}, a_{i}^{(1)}, a_{i}^{(2)}, \ldots \quad \delta_{i}=a_{i}^{(v+1)}-a_{i}^{(v)} .
$$

The outcome of a measurement of the $r$ physical quantities which are described microscopically by phase functions $A_{1}(x), \ldots, A_{r}(x)$ therefore yields the information that the phase point $x$ corresponding to the system lies in a certain domain

$$
\left\{x \in \Sigma \mid a_{i}^{\left(v_{i}\right)} \leqslant A_{i}(x) \leqslant a_{i}^{\left(v_{i}+1\right)}, i=1, \ldots, r\right\} ;
$$

the better the accuracy of the measuring instruments is, the smaller will be (3.7). The set of these domains obviously fulfils the conditions (3.2)-(3.4).

This introduction of phase cells, however, is still not unique for two reasons, although one would intuitively expect this nonuniqueness not to be relevant:

(i) The accuracy of a measuring instrument is itself not precisely measurable, for it is an estimate based on arguments of probability theory. 
(ii) If (3.6) is a suitable partition of the range of $A_{i}$, so will be $b_{i}^{\left(v_{i}\right)}:=a_{i}^{\left(v_{i}\right)}+\varepsilon$ with any $\varepsilon \in \mathbf{R}$ sufficiently small. We shall come back to this problem in section 4.6.

The physical justification of the use of the coarse-grained distribution $P(x ; t)$ with the cells $(3.7)$ instead of $\varrho(x ; t)$ lies in the fact that all information which is accessible for the macroscopic observer about $\varrho(x ; t)$ is contained in $P(x ; t)$. Consequently, expectation values have to be defined as

$$
\left\langle A_{i}\right\rangle(t):=\bigcup_{\underline{j}} P(x ; t) A_{i}(x) \mathrm{d} \mu=\sum_{j} P_{j}(t) \int_{\varrho_{j}} A_{i}(x) \mathrm{d} \mu
$$

and the entropy functional as

$$
S_{\mathrm{cg}}(t):=-k \int_{2} P(x ; t) \ln P(x ; t) \mathrm{d} \mu=-k \sum_{j} \mu\left(\Omega_{j}\right) P_{j}(t) \ln P_{j}(t),
$$

which is called the coarse-grained entropy.

\section{Properties of the coarse-grained entropy}

4.1. Postulates (P1) and (P2) are trivially fulfilled.

4.2. In equilibrium situations, $S_{\mathrm{cg}}$ reproduces all thermodynamic properties of $S$.

This is due to the identity $g(x)=P(x)$ for the microcanonical distribution which implies that the coarse-grained entropy is identical with the Gibbs entropy $S_{\mathrm{G}}$ for equilibrium. It is well-known that the Gibbs entropy has all thermodynamic properties of $S$ (e.g. ref. 2). Thus, postulate (P3) is fulfilled.

The following subsections shall deal with the question whether postulate (P4) is fulfilled.

4.3. For $t \rightarrow \infty$, we get the Second Law.

Putting $f(x)=\chi\left(\Omega_{j}\right)$ in (2.5) where $\chi$ is the characteristic function, we obtain

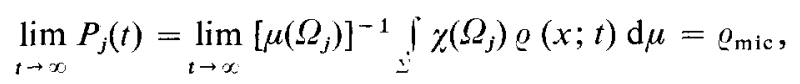

i.e. $P(x ; t)$ converges pointwise to $\varrho_{\text {mic }}$. This implies

$$
\begin{aligned}
\lim _{t \rightarrow \infty} S_{\mathrm{cg}}(t) & =-k \lim _{t \rightarrow \infty} \sum_{j} \mu\left(\Omega_{j}\right) P_{j}(t) \ln P_{j}(t) \\
& =k \int_{-} Q_{\mathrm{mic}} \ln Q_{\mathrm{mic}} \mathrm{d} \mu=S(\mathscr{B})
\end{aligned}
$$

The Second Law follows directly from the variational property of the entropy functional (3.9): it is maximized by a function which is constant in $\Sigma$ (in comparison to all $f \in L_{2}(\Sigma, \mu)$ with the same normalization) (e.g. ref. 22). 
This shows that $S_{\mathrm{cg}}(t)$ reaches $S(\mathscr{B})$ for $t \rightarrow \infty$ but we do not know yet whether the approach of $S_{\mathrm{cg}}(t)$ to its equilibrium value takes place well-timed in the sense of postulate (P4). In the literature, estimates on the time within which $P(x ; t)$ reaches stationary values (or equivalently, $S_{\mathrm{cg}}(t)$ reaches approximately $S(\mathscr{B})$ ) range from the order of many recurrence times ${ }^{19}$ ) to the relaxation time of the system $^{23,24}$ ). Clearly, a definite decision whether (P4) is fulfilled involves the comparison of the time after which $S_{\mathrm{cg}}(t)$ for a particular physical situation is approximately equal to $S(\mathscr{B})$, and the measured relaxation time $\tau_{\text {rel }}$. In the next section, we shall compare the time after which $S_{\mathrm{cg}}(t)$ has approximately reached $S(\mathscr{B})$ with the predicted relaxation time $\left\langle\tau_{\mathrm{rel}}\right\rangle$ : this is the time after which the slowest relaxing expectation value $\left\langle A_{i}\right\rangle(t)$ has reached its new equilibrium value within the given accuracy. This procedure is only a check on the internal consistency of the theory.

4.4. $S_{\mathrm{cg}}(t)$ tends to the new equilibrium value $S(\mathscr{B})$ in general faster than those expectation values $\left\langle A_{i}\right\rangle(t)$ which are not constant in time, to their new equilibrium values

$$
\left\langle A_{i}\right\rangle_{\mathrm{eq}}:=\lim _{t \rightarrow \infty} \int_{\Sigma} P(x ; t) A_{i}(x) \mathrm{d} \mu=o_{\mathrm{mic}} \int_{\Sigma} A_{i}(x) \mathrm{d} \mu .
$$

Because of the convergence of the $P_{f}(t)$ to $g_{\text {mic }}$ (eq. (4.1)), we may write

$$
P_{j}(t)=\varrho_{\mathrm{mic}}+\varepsilon_{j}(t),
$$

with

$$
\lim _{t \rightarrow \infty} \varepsilon_{j}(t)=0 \quad \text { for all } j
$$

and

$$
\sum_{j} \mu\left(\Omega_{j}\right) \varepsilon_{j}(t)=0 .
$$

(4.6) is seen by integrating (4.4) over $\Sigma$.

Substituting (4.4) into (3.8) and using (4.3), we get

$$
\left\langle A_{i}\right\rangle(t)-\left\langle A_{i}\right\rangle_{\mathrm{eq}}=\sum_{j} \varepsilon_{j}(t) \int_{\Omega_{j}} A_{i}(x) \mathrm{d} \mu ;
$$

substitution of (4.4) into (3.9) yields

$$
\begin{aligned}
S_{\mathrm{cg}}(t)= & -k \int_{\Sigma} \varrho_{\mathrm{mic}} \ln \varrho_{\mathrm{mic}} \mathrm{d} \mu \\
& -k \sum_{j} \mu\left(\Omega_{j}\right)\left[\varrho_{\mathrm{mic}}+\varepsilon_{j}(t)\right] \ln \left[1+\varepsilon_{j}(t) / \varrho_{\mathrm{mic}}\right],
\end{aligned}
$$


where we used (4.6). For $t$ large enough such that

$$
\left|\varepsilon_{j}(t)\right| / \underline{o m i c}_{\mathrm{mic}}<1 \quad \text { for all } j
$$

i.e. not too far from the final equilibrium state, wc may use the cxpansion

$$
\ln (1+x)=x-x^{2} / 2+x^{3} / 3 \cdots, \quad-1<x \leqslant 1,
$$

yielding for (4.8) with (4.6)

$$
S_{\mathrm{cg}}(t)-S(\mathscr{B})=-\left(k / 2 \varrho_{\mathrm{mic}}\right) \sum_{j} \varepsilon_{j}^{2}(t) \mu\left(\Omega_{j}\right)+\mathcal{O}\left(\varepsilon_{j}^{3}\right)
$$

Thus, $\left(\left\langle A_{i}\right\rangle(t)-\left\langle A_{i}\right\rangle_{\mathrm{eq}}\right)$ goes to zero linearily in the $\varepsilon_{j}(t)$, whereas $\left(S_{\mathrm{cg}}(t)-S(\mathscr{B})\right)$ falls off quadratically in the $\varepsilon_{j}(t)$ (eqs. (4.7) and (4.11)).

There is the mathematical possibility, however, that as a function of $t,(4.7)$ decays faster than (4.11). This may happen if the terms in the sum (4.7) which contain the slowest decaying $\varepsilon_{j_{1}}(t), \ldots, \varepsilon_{j_{k}}(t)$ either vanish or cancel each other, and the remaining terms decay faster than

$$
-\left(k / 2 \varrho_{\mathrm{mic}}\right) \sum_{n=j_{1}}^{j_{k}} \varepsilon_{n}^{2}(t) \mu\left(\Omega_{j}\right),
$$

which is the slowest decaying part of (4.11). But if there is at least one $A_{i}(x)$ for which these cancellations do not occur, we may expect that a modified version of (P4) holds where $\tau_{\text {rel }}$ is replaced by $\left\langle\tau_{\text {rel }}\right\rangle$. The next property of $S_{\mathrm{cg}}(t)$, however, raises new problems.

4.5. $S_{\mathrm{cg}}(t)$ is lowered for a refinement of the cells ( $t$ fixed).

Let $\left\{\Omega_{j} \mid j \in J\right\}$ be a set of phase cells, and let $\left\{\Omega_{j}^{m} \mid m=1, \ldots, m_{j} ; j \in J\right\}$ be a refinement of $\left\{Q_{j}\right\}$, i.e.

$$
\begin{aligned}
& \bigcup_{m=1}^{m_{J}} \Omega_{j}^{m}=\Omega_{j}, \\
& \mu\left(\Omega_{j}^{m}\right)>0
\end{aligned}
$$

and

$$
\sum_{m=1}^{m_{j}} \mu\left(\Omega_{j}^{m}\right)=\mu\left(\Omega_{j}\right)
$$

With the notation

$$
P_{j}^{m}(t):=\left[\mu\left(\Omega_{j}^{m}\right)\right]^{-1} \int_{\Omega_{j}^{m}} \varrho(x ; t) \mathrm{d} \mu,
$$


the following identity holds:

$$
\mu\left(\Omega_{j}\right) P_{j}=\sum_{m} \mu\left(\Omega_{j}^{m}\right) P_{j}^{m},
$$

since both sides of (4.16) are equal to $\int_{\Omega_{j}} \varrho(x ; t) \mathrm{d} \mu$. With $S_{\mathrm{cg}}$ corresponding to the partition $\left\{\Omega_{j}\right\}$ and $\bar{S}_{\mathrm{cg}}$ to $\left\{\Omega_{j}^{m}\right\}$, we get

$$
\begin{aligned}
S_{\mathrm{cg}}-\bar{S}_{\mathrm{cg}} & =-k\left\{\sum_{j} \mu\left(\Omega_{j}\right) P_{j} \ln P_{j}-\sum_{j, m} \mu\left(\Omega_{j}^{m}\right) P_{j}^{m} \ln P_{j}^{m}\right\} \\
& =-k\left\{\sum_{j, m} \mu\left(\Omega_{j}^{m}\right)\left[P_{j}^{m} \ln P_{j}-P_{j}^{m} \ln P_{j}^{m}\right]\right\} \\
& \geqslant-k\left\{\sum_{j, m} \mu\left(\Omega_{j}^{m}\right)\left[P_{j}-P_{j}^{m}\right]\right\}=0,
\end{aligned}
$$

where we used successively (4.16), then the well-known inequality (e.g. ref. 22)

$$
x \ln y-x \ln x \leqslant y-x
$$

and finally (4.14) and (4.16) ${ }^{\ddagger}$. Equality in (4.17) holds only for $P_{j}=P_{j}^{m} ; m$ $=1, \ldots, m_{j} ; j \in J$.

Inequality (4.17) may be intuitively understood by the fact that $\bar{P}(x, t)$ (corresponding to $\left.\left\{\Omega_{j}^{m}\right\}\right)$ is a better approximation to $\varrho(x ; t)$ than $P(x ; t)$ and that

$$
S_{\mathrm{G}}=-k \int_{\Sigma} \varrho(x ; t) \ln \varrho(x ; t) \mathrm{d} \mu
$$

has still its low initial value

$$
-k \int_{\Sigma} \varrho(x ; 0) \ln \varrho(x ; 0) \mathrm{d} \mu .
$$

We prove that $\bar{P}(x ; t)$ is a better approximation to $\varrho(x ; t)$ than $P(x ; t)$ by calculating

$$
\begin{aligned}
\int_{\Omega_{j}} & {[P(x ; t)-\varrho(x ; t)]^{2} \mathrm{~d} \mu-\int_{\Omega_{j}}[\bar{P}(x ; t)-\varrho(x ; t)]^{2} \mathrm{~d} \mu } \\
& =\sum_{m} \int_{\Omega_{j}^{m}}\left[\left(P_{j}\right)^{2}-2 P_{j} \varrho-\left(P_{j}^{m}\right)^{2}+2 P_{j}^{m} \varrho\right] \mathrm{d} \mu \\
& =\sum_{m} \mu\left(\Omega_{j}^{m}\right)\left[P_{j}-P_{j}^{m}\right]^{2} \geqslant 0 .
\end{aligned}
$$

The property (4.17) has led to the opinion that the time it takes $P(x ; t)$ to become approximately uniform (or equivalently $S_{\mathrm{cg}}(t)$ to reach approximately

* A different proof has been given recently by Liboff ${ }^{32}$ ). 
$S(\mathscr{B})$ ) depends essentially on the size of the cells ${ }^{25,26}$ ). From this, one may get the impression that (P4) cannot be fulfilled for the following reason: Experimentally, the time behavior of the thermodynamic quantities remains almost unaffected by an increase of the accuracies of the measuring instruments (unless the starting accuracy was extremely low and unless previously irrelevant physical effects become relevant due to the increase of the accuracy). This implies that the relaxation time is stable against variations of the experimental accuracy for a fixed set of measuring instruments from certain accuracies on. If, on the other hand, the time it takes $S_{\mathrm{cg}}(t)$ to reach approximately $S(\mathscr{B})$ can be made longer and longer by an increase of the experimental accuracy, a region where it exceeds $\tau_{\text {rel }}$ may be attained, in conflict with (P4). (This implies, by the way, that the predicted relaxation time $\left\langle\tau_{\text {rel }}\right\rangle$ is longer than the actual relaxation time $\tau_{\text {rel }}$ according to section 4.4.) Therefore, we would like to have the same stability property for $S_{\mathrm{cg}}(t)$ as for $\tau_{\mathrm{rel}}$.

4.6. For fixed $A_{1}(x), \ldots, A_{r}(x)$ and $t$, and arbitrarily varying inaccuracies, an upper and a lower bound on $S_{\mathrm{cg}}(t)$ exist; for $t \rightarrow \infty$, the lower bound converges towards the upper bound.

The upper bound on $S_{\mathrm{cg}}(t)$ is easily found to be

$$
S_{\mathrm{ce}}(t) \leqslant-k \prod_{i} Q_{\mathrm{mic}} \ln Q_{\mathrm{mic}} \mathrm{d} \mu \quad \text { for all }\left\{\Omega_{j}\right\}
$$

because of the variational property of the entropy functional discussed in section 4.3. By "for all $\left\{\Omega_{j}\right\}$ " we mean in this section all sets of phase cells corresponding to fixed $A_{1}(x), \ldots, A_{r}(x)$ as introduced in section 3 , with arbitrary inaccuracies.

For the lower bound, we use the result of section 4.5 that $S_{\mathrm{cg}}(t)$ is lowered for a refinement of the cells. We thus get the lower bound as

$$
\lim _{\substack{\mu\left(\Omega_{j}\right) \rightarrow 0 \\ \text { a } 11 j}} S_{\mathrm{cg}}(t)
$$

if this limit exists. The limit $\mu\left(\Omega_{j}\right) \rightarrow 0$ in (4.21) has to be taken by means of $\delta_{i} \rightarrow 0, i=1, \ldots, r$; this corresponds to exact measurements of the $r$ physical quantities $A_{i}^{\text {exp }}$. We first consider

$$
P^{(x)}(x ; t):=\lim _{\substack{\delta_{i} \rightarrow 0 \\ i=1, \ldots, r}} P(x ; t),
$$

or, explicitly

$$
\begin{aligned}
\left.P^{(\infty)}(x ; t)\right|_{x \in \Omega^{(-r)}} & =\lim _{\substack{\delta_{i} \rightarrow 0 \\
i=1, \ldots, r}}\left(\int_{\substack{a_{i} \leqslant A_{i}(x) \leqslant a_{i}+\delta_{i} \\
i=1, \ldots, r}}^{j} g(x ; t) \mathrm{d} \mu / \int_{\substack{a_{i} \leqslant A_{i}(x) \leqslant a_{i}+\delta_{i} \\
i=1, \ldots, r}} \mathrm{~d} \mu\right),
\end{aligned}
$$


where

$$
\Omega^{(-r)}:=\left\{x \in \Sigma \mid A_{i}(x)=a_{i} ; i=1, \ldots, r\right\} .
$$

The superscript $(-r)$ at $\Omega^{(-r)}$ shall denote that by taking the limits $\delta_{i} \rightarrow 0$ the dimensionality of $\Omega$ has been diminuished by $r$; clearly

$$
\mu\left(\Omega^{(-r)}\right)=0 .
$$

For the calculation of (4.23), we use

$$
\begin{aligned}
& \int_{\substack{a_{i} \leqslant A_{i}(x) \leqslant a_{i}+\delta_{i} \\
i=1, \ldots, r}} f(x) \mathrm{d} \mu \\
= & \frac{\partial^{r}}{\partial a_{1} \cdots \partial a_{r}} \int_{\substack{A_{i}(x) \leqslant a_{i} \\
i=1, \ldots, r}} f(x) \mathrm{d} \mu \prod_{i=1}^{r} \delta_{i}+\mathcal{O}\left(\delta_{i}^{2}\right)
\end{aligned}
$$

which may be seen either by induction for $r$ or by introducing new variables such that

$$
\mathrm{d} \mu=\mathrm{d} A_{1} \cdots \mathrm{d} A_{r} \mathrm{~d} \mu^{(-r)} .
$$

Using (4.26) for $f=\varrho$ and $f=1$, we obtain for (4.23)

$$
\begin{aligned}
\left.P^{(\infty)}(x ; t)\right|_{x \in \Omega^{(-r)}} & \frac{\partial^{r}}{\partial a_{1} \cdots \partial a_{r}} \int_{\substack{A_{i}(x) \leqslant a_{l} \\
i=1, \ldots, r}} \varrho(x ; t) \mathrm{d} \mu / \frac{\partial^{r}}{\partial a_{1} \cdots \partial a_{r}} \int_{\substack{A_{i}(x) \leqslant a_{i} \\
i=1, \ldots, r}} \mathrm{~d} \mu .
\end{aligned}
$$

Obviously, $P^{(\infty)}(x ; t)$ is in general an extremely crude approximation to $\varrho(x ; t)$ since it smears $\varrho(x ; t)$ out on the sets $\Omega^{(-r)}$ which are of dimensionality $\operatorname{dim} \Sigma-r$ where $\operatorname{dim} \Sigma$ is of the order $10^{23}$ and $r$ about 10 or 100 . We may therefore expect that $P^{(\infty)}(x ; t)$ behaves very much like $P(x ; t)$.

Because $\varrho(x ; t)$ is uniformly bounded (eq. (2.3)) all $P(x ; t)$ are uniformly bounded which implies

$$
|P(x, t) \ln P(x, t)| \leqslant 1+B \ln B \text { for all }\left\{\Omega_{j}\right\} \text { and all } t .
$$

For the calculation of (4.21) we may then apply the theorem of Lebesgue yielding

$$
S_{\mathrm{cg}}^{(\infty)}(t):=\lim _{\substack{\delta_{i} \rightarrow 0 \\ i=1, \ldots, r}} S_{\mathrm{cg}}(t)=-k \int_{\underline{-}} P^{(\infty)}(x ; t) \ln P^{(\infty)}(x ; t) \mathrm{d} \mu,
$$


with

$$
S_{\mathrm{cg}}^{(\infty)}(t) \leqslant S_{\mathrm{cg}}(t) \quad \text { for all }\left\{\Omega_{j}\right\} \text { and all } t .
$$

In order to discuss the long time behavior of this lower bound $S_{\mathrm{cg}}^{(\infty)}(t)$ we want to calculate

$$
\lim _{t \rightarrow \infty} \frac{\partial^{r}}{\partial a_{1} \cdots \partial a_{r}} \int_{\substack{A_{i}(x) \leqslant a_{i} \\ i=1, \ldots, r}} \varrho(x ; t) \mathrm{d} \mu,
$$

which is the numerator of (4.28). If we are allowed to interchange $\lim _{t \rightarrow \infty}$ with $\hat{o}^{r} / \partial a_{1} \cdots \partial a_{r}$ in (4.32), we get

$$
\lim _{t \rightarrow \infty} P^{(\infty)}(x ; t)=\varrho_{\text {mic }},
$$

because of the assumed weak convergence of $\varrho(x ; t)$ to the microcanonical distribution (eq. (2.5)). The interchange of $\lim _{t \rightarrow \infty}$ with $\partial^{r} / \partial a_{1} \cdots \partial a_{r}$ may be forbidden in pathological cases, for instance if the thin filaments in which $\varrho(x ; t)$ is stretched keep running parallel to one of the hypersurfaces $A_{i}(x)=a_{i}$. But since the $A_{i}(x)$ are macroscopic quantities and their number is exceedingly smaller than the dimensionality of $\Sigma$, we may assume that these filaments intersect with the hypersurfaces randomly enough to permit the interchange. This assumption may be also looked upon in the following way. The coarse-graining process, i.e. the integration over all coordinates and momenta turns the weak convergence of $\varrho(x ; t)$ into pointwise convergence of $P(x ; t)$. Leaving out integration over $r$ variables shall have the effect that the resulting function $P^{(\infty)}(x ; t)$ still converges to $\varrho_{\text {mic }}$, at least almost everywhere. Sufficient for this is the condition that $\varrho(x ; t)$ converges weakly to $\varrho_{\text {mic }}$ not only with respect to the original measure $\mu$, but also with respect to the "reduced" measure $\mu^{(-r)}$ introduced in (4.27).

Since inequality (4.29) holds for $P^{(\infty)}(x ; t)$ as well, we get with (4.33)

$$
\lim _{t \rightarrow \infty} S_{\mathrm{cg}}^{(\infty)}(t)=-k \int_{\Sigma} \varrho_{\text {mic }} \ln \varrho_{\text {mic }} \mathrm{d} \mu .
$$

We combine the results (4.20), (4.31) and (4.34) in the following lemma.

Lemma. For a given $\varepsilon>0$, there exists a time $\tau_{0}$ such that for all $t \geqslant \tau_{0}$ the following inequality holds

$$
\begin{gathered}
-k \int_{\Sigma} \varrho_{\mathrm{mic}} \ln \varrho_{\mathrm{mic}} \mathrm{d} \mu-\varepsilon \leqslant S_{\mathrm{cg}}(t) \leqslant-k \int_{\Sigma} \varrho_{\mathrm{mic}} \ln \varrho_{\mathrm{mic}} \mathrm{d} \mu \\
\text { for all }\left\{\Omega_{j}\right\} \text { with fixed } A_{1}(x), \ldots, A_{r}(x) .
\end{gathered}
$$


The significance of this result is firstly that $S_{\mathrm{cg}}(t)$, and thus the time within which it reaches its equilibrium value within a certain inaccuracy, gets the more insensitive against variations of the cells the smaller the cells are. This is precisely the stability condition on a microscopic expression for the entropy discussed at the end of the previous section. Secondly, the dependence of $S_{\mathrm{cg}}(t)$ on the set of cells in general becomes less and less relevant as time goes on. Thus, the remaining nonuniqueness of the choice of the cells (section 3 ) is unimportant at least for sufficiently large times. We may further conclude that a sufficient condition for the fulfilment of postulate (P4) is

$$
\tau_{0} \leqslant \tau_{\text {rel }} .
$$

In the next section, we shall give an indication about the relation between $\tau_{\text {rel }}$ and $\tau_{0}$.

4.7. The times $\tau_{\mathrm{rel}}, \tau_{0}$, and the time after which some $S_{\mathrm{cg}}(t)$ reaches approximately $S(\mathscr{B})$ qualitatively depend on the macroscopic variables in the same way.

The qualitative dependence of $\tau_{\text {rel }}$ on the $A_{i}$ is obvious; adding new variables can never lower $\tau_{\text {rel }}$. The same holds for the two other times, since further functions $A_{r+1}(x), \ldots, A_{R}(x)$ induce a subpartition of the original cells. Then, section 4.5 yields the desired result.

\section{A model calculation}

In this section, we want to illustrate the results of the previous section by treating explicitely a simple model system. The system consists of one classical point particle of mass $m$ enclosed in a one-dimensional box of length $L$ with perfectly reflecting walls; inside the box, the particle moves freely. Although this model is exceedingly simple, it has served quite well to illustrate several aspects of statistical mechanics (e.g. refs. 27, 28 and references cited therein and ref. 16).

A particle with energy not larger than $b^{2} / 2 m, b>0$, is described in an energy shell $\Sigma$ consisting of all points $(q, p) \in \mathbf{R}^{2}$ with $q \in[0, L]$ and $p \in[-b, b]$. With the invariant measure $\mathrm{d} \mu=\mathrm{d} q \mathrm{~d} p$ we have

$$
\mu(\Sigma)=\int_{-b}^{b} \mathrm{~d} p \int_{0}^{L} \mathrm{~d} q=2 b L .
$$

We incorporate the reflecting walls by reflecting boundary conditions (e.g. ref.29). For $t=0$ we impose the initial condition that the particle shall be in a certain part of the box on the left hand side:

$$
\begin{aligned}
& =\text { const } \text { for } 0 \leqslant q \leqslant g L, \\
\left.\varrho(q, p ; t \leqslant 0)\right|_{(q, p) \in \Sigma} & =0 \text { for } g L<q \leqslant L,
\end{aligned}
$$


with $0<g<1$. Using normalization to unity and the reflecting boundary conditions we obtain

$$
\begin{aligned}
\varrho(q, p ; t \leqslant 0)= & (2 b g L)^{-1} \theta(b+p) \theta(b-p) \\
& \times \sum_{i \in \mathbf{Z}} \theta(q+g L-2 i L) \theta(g L+2 i L-q)
\end{aligned}
$$

in fulfilment of assumption 1 of section 2. Since the hamiltonian consists only of the kinetic part, the solution of the Liouville equation is given by (e.g. ref. 30)

$$
\varrho(q, p ; t)=\varrho(q-p t / m, p ; 0) .
$$

Inserting (5.3) into (5.4) yields for $t>0$

$$
\begin{aligned}
\varrho(q, p ; t)= & (2 b g L)^{-1} \theta(p / b+1) \theta(1-p / b) \\
& \times \sum_{i \in \mathbf{Z}} \theta(p / b-(m L / b t)(q / L-g-2 i)) \\
& \times 0((m L / b t)(q / L+g-2 i)-p / b) .
\end{aligned}
$$

We prefer the representation (5.5) of the solution of the Liouville equation to a Fourier series representation as, for instance, in ref. 31 since the latter is difficult to handle in numerical calculations. Expression (5.5) consists for finite $t$ only of a finite number of nonvanishing terms in the original domain $\Sigma$ and may therefore be calculated exactly.

$\varrho(q, p ; t)$ converges weakly to $\left.{ }^{22}\right)$

$$
\varrho_{\mathrm{eq}}(p)=(2 L)^{-1} \int_{0}^{2 L} \varrho(q, p ; 0) \mathrm{d} q,
$$

which yields with (5.3)

$$
\varrho_{\mathrm{cq}}(p)=(2 b I)^{-1} \theta(b+p) \theta(b-p)=(2 h I)^{-1} \theta(b-|p|),
$$

which is the microcanonical distribution in $\Sigma$; assumption 2 is therefore met.

For the introduction of phase cells, we assume that we are able to measure the position of the particle with a certain inaccuracy $\delta$ that shall be independent of the position of the particle. Following section 3, we have as "macroscopic" quantity

$$
A(q, p)=q .
$$

The range of $A$, i.e. the interval $[0, L]$, is then subdivided into subintervals of equal length $\delta$. This yields the corresponding cells

$$
\Omega_{j}=\{(q, p) \in \Sigma \mid(j-1) \delta \leqslant q \leqslant j \delta\}, \quad j=1, \ldots, z,
$$


where $z$ is the number of cells; we chose $\delta$ such that $L$ is a multiple of $\delta$, i.e.,

$$
z \delta=L, \quad z \in \mathbf{N} .
$$

For the coarse-grained distribution function (3.5), we get with (5.5) and (5.10)

$$
\begin{aligned}
P_{j}(t)= & \left(\int_{(j-1) \delta}^{j \delta} \mathrm{d} q \int_{-b}^{b} \mathrm{~d} p\right)^{-1} \int_{(j-1) \delta}^{j \delta} \mathrm{d} q \int_{-b}^{b} \mathrm{~d} p \varrho(q, p ; t) \\
= & (z / 4 b g L) \int_{(j-1) / z}^{j / z} \mathrm{~d} x \int_{-1}^{1} \mathrm{~d} y \sum_{i \in \mathrm{Z}} \theta(y-(x-g-2 i) / \tau) \\
& \times \theta((x+g-2 i) / \tau-y),
\end{aligned}
$$

where we introduced dimensionless variables

$$
x:=q / L, \quad y:=p / b, \quad \text { and } \quad \tau:=b t / m L .
$$

One can easily see that in the sum of integrands in (5.11) only those terms are different from zero inside of $\Sigma$, for which the index $i$ fulfils the inequality

$$
(-g-\tau) / 2<i<(1+g+\tau) / 2
$$

i.e. the sum is actually finite. For $t \rightarrow \infty$, we get with (5.7)

$$
\lim _{t \rightarrow \infty} P_{j}(t)=(2 b L)^{-1} .
$$

With the aid of (5.13), the $P_{j}(t)$ may be easily evaluated by means of a small computer. Finally for the coarse-grained entropy, we get according to (3.9)

$$
S_{\mathrm{cg}}(t)=-(2 b L / z) \sum_{j=1}^{z} P_{j}(t) \ln P_{j}(t),
$$

where we have put $k=1$. The time limit of $S_{\mathrm{cg}}(t)$ is according to (4.2)

$$
\lim _{t \rightarrow \infty} S_{\mathrm{cg}}(t)=\ln (2 b L)=\ln \mu(\Sigma),
$$

as expected.

For the actual computation of $S_{\mathrm{cz}}(t)$, we put

$$
m=L=b=1 ;
$$

this is no loss of generality as may be seen from (5.11) and (5.15) since those parameters enter the expression for $P_{j}$ as a function of $\tau$ only as factors. Fig. 1 shows 
$S_{\mathrm{cg}}(t)$ for an initial width $g=0.5$ and different cell numbers (to $z=\infty$ we shall come back later); the graphs of fig. 1 and of the following figures have been obtained by interpolation of exactly calculated points of distance $\Delta t=\frac{1}{16}$.
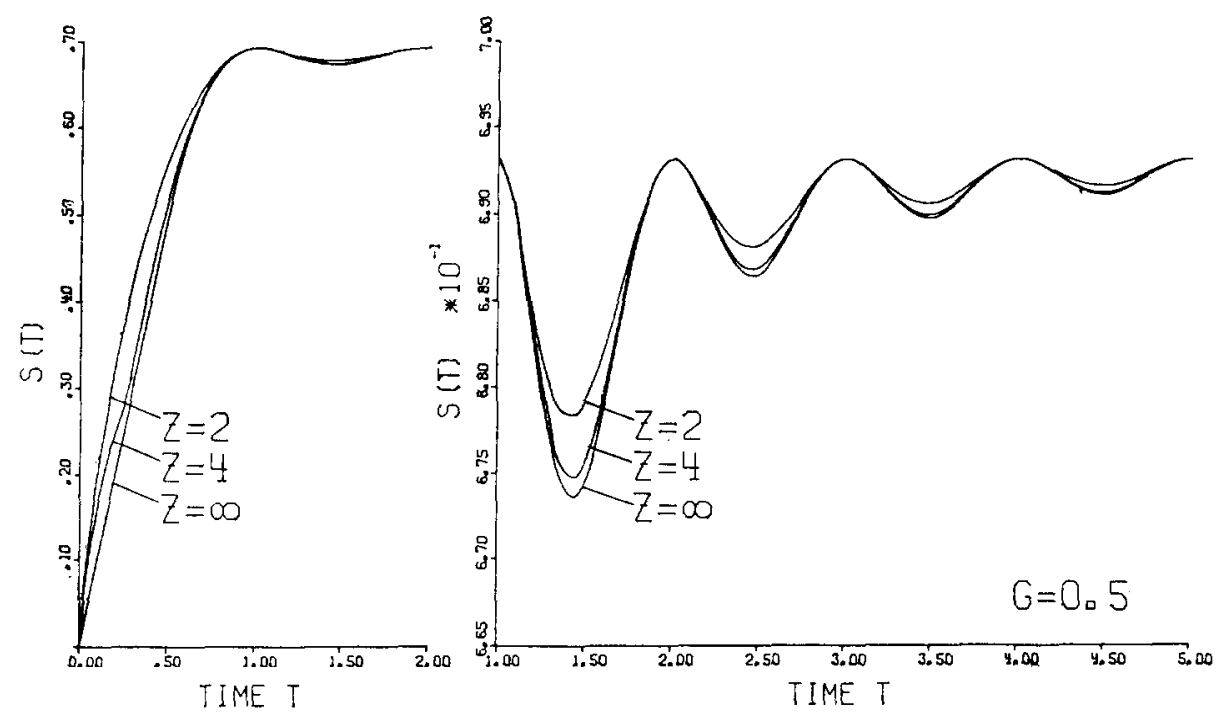

Fig. 1

We have a nonmonotonic approach of $S_{\mathrm{cg}}(t)$ to its maximum value; due to the geometry of the problem, for $\tau=n, n \in \mathbf{N}, S_{\mathrm{cg}}(t)$ takes its equilibrium value. Runs with different values for $g$ look typically the same as the one for $g=0.5$. The relative minima in fig. 1 are near $\tau=1.5,2.5, \ldots$ Using the geometrical properties of the problem, one can derive a formula for $S_{\mathrm{cg}}(t)$ for these points by evaluating (5.11). The result for $g=0.5$ and $z=2$ is

$$
\begin{aligned}
S_{\mathrm{cg}}(t)= & \ln (2 \tau)-[(\tau+0.25) \ln (\tau+0.25) \\
& +(\tau-0.25) \ln (\tau-0.25)] /(2 \tau) \text { for } \tau=0.5,1.5, \ldots,
\end{aligned}
$$

which yields for large times

$$
S_{\mathrm{cg}}(t) \approx \ln 2-\tau^{-2 / 32} .
$$

For the expectation value of $q$, we get according to definition (3.8)

$$
\begin{aligned}
\langle q\rangle(t) & =\int_{0}^{L} \mathrm{~d} q \int_{-b}^{b} \mathrm{~d} p q P(q, p ; t)=2 b \sum_{j=1}^{z} P_{j}(t) \int_{(j-1) \delta}^{j \delta} q \mathrm{~d} q \\
& =\left(2 b L^{2} / z^{2}\right) \sum_{j=1}^{z} P_{j}(t)(j-0.5),
\end{aligned}
$$




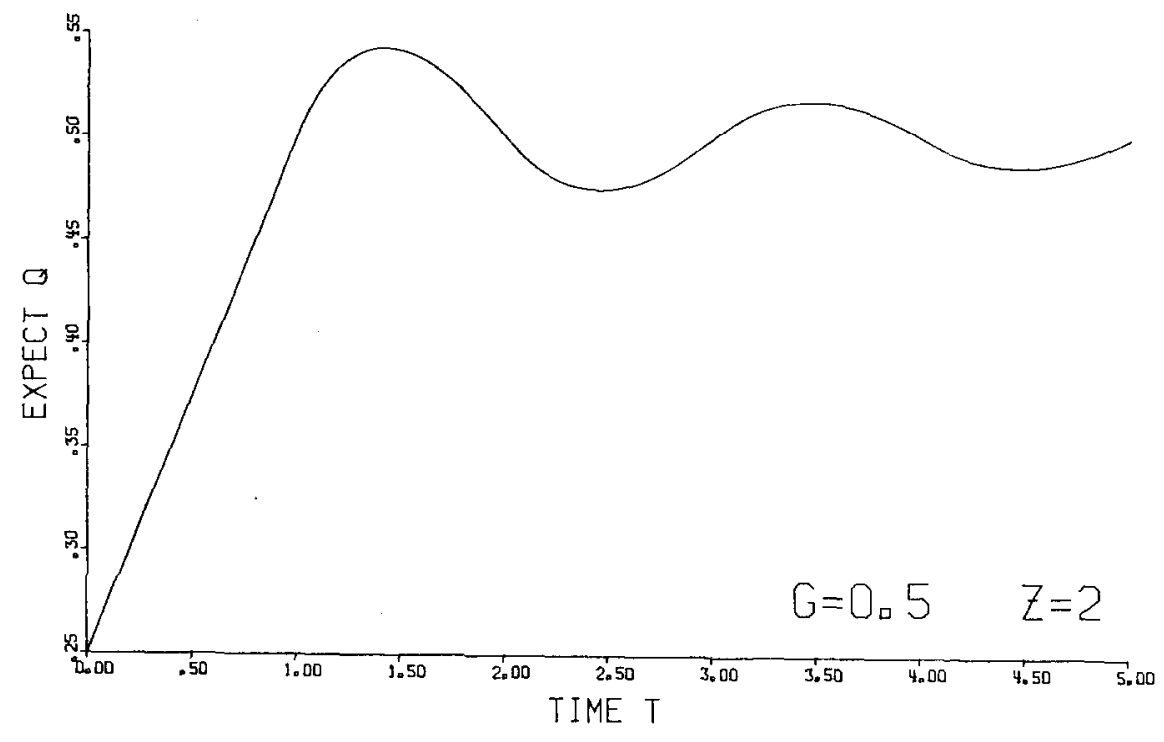

Fig. 2

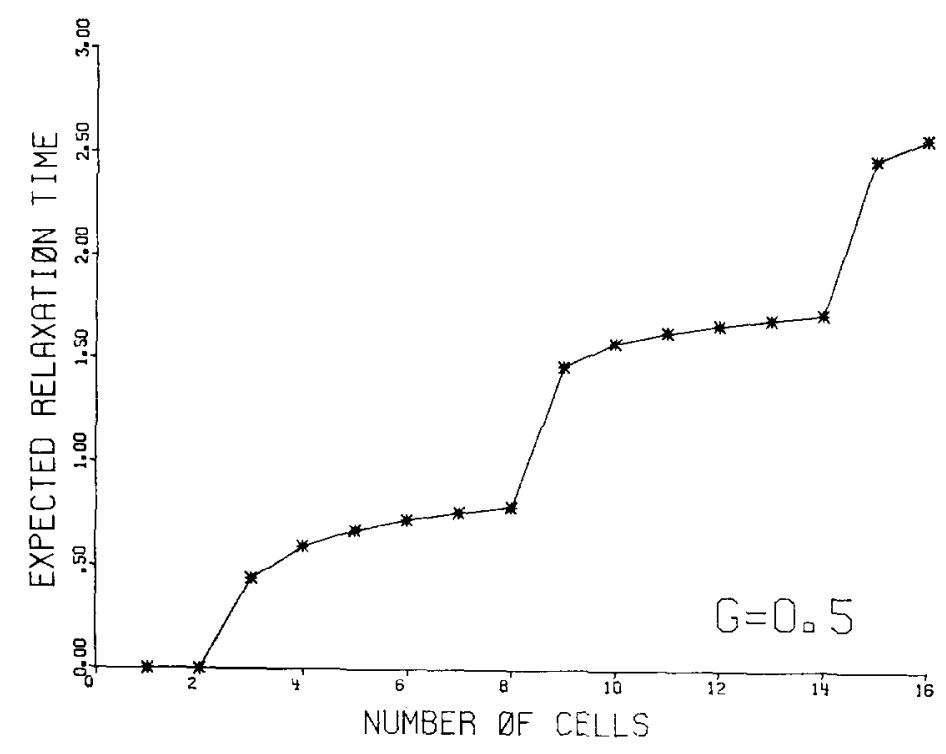

Fig. 3 
with the equilibrium value

$$
\langle q\rangle_{\mathrm{eq}}=L / 2 .
$$

A plot of $\langle q\rangle(t)$ for $g=0.5$ and $z=2$ is shown in fig. 2 .

Evaluating (5.20) directly for the same parameters yields for the same $\tau$ 's as in (5.18) (with $m=L=b=1$ )

$$
\langle q\rangle(t)=0.5 \mp \tau^{-1} / 16 \text { for } \tau=\left\{\begin{array}{l}
0.5,2.5,4.5, \ldots \\
1.5,3.5,5.5, \ldots
\end{array}\right.
$$

Now we investigate the dependence of $\left\langle\tau_{\mathrm{rel}}\right\rangle$ on the number of cells. For the model, $\left\langle\tau_{\text {rel }}\right\rangle$ is the time after which

$$
|\langle q\rangle(t)-0.5| \leqslant 1 / 2 z \quad \text { for } \quad t \geqslant\left\langle\tau_{\text {rel }}\right\rangle
$$

holds, i.e. with the accuracy $1 / z$ given, $\langle q\rangle(t)$ cannot further be distinguished from its equilibrium value. A plot of $\left\langle\tau_{\mathrm{rel}}\right\rangle v s$. the number of cells for $g=0.5$ is given in fig. 3.

$\left\langle\tau_{\text {rel }}\right\rangle$ gets almost stable between 5 and 8 cells; with further increase of the accuracy, the slowly decaying fluctuations of $\langle q\rangle(t)$ are detected. The stability region is fairly small since the difference of $\langle q\rangle$ due to the change of the state is only about five times larger than the amplitude of the first fluctuation; in a more physical situation, however, these two quantities may differ by some orders of magnitude.

Next, we want to check the property of $S_{\mathrm{cg}}(t)$ to reach its equilibrium value faster than an expectation value, as stated in section 4.4. To this end, we calculate

$$
Q(t):=\left[S_{\mathrm{cg}}(t)-S_{\mathrm{cg}}(\infty)\right] /\left[\langle q\rangle(t)-\langle q\rangle_{\mathrm{eq}}\right]^{2},
$$

which function must neither approach zero nor infinity for $t \rightarrow \infty$. For the special $\tau$ values $0.5,1.5, \ldots$ this is seen by (5.19) and (5.22); plots of $Q(t)$ are given in figs. 4 and 5. For $z=2, Q(t)$ approaches a constant, for $z=4, Q(t)$ approaches a periodic function, respectively; both cases agree with the result of section 4.4.

The property of $S_{\mathrm{cg}}(t)$ to decrease for a refinement of the cells (section 4.5 ) is seen in fig. 1. The calculation of the lower bound (i.e. $z=\infty$ in fig. 1) follows section 4.6: $P^{(\infty)}(q, p ; t)$ is according to $(4.28)$

$$
\begin{aligned}
P^{(\infty)}(q ; t) & =\frac{\partial}{\partial q} \int_{0}^{q} \mathrm{~d} q^{\prime} \int_{-b}^{b} \mathrm{~d} p \varrho\left(q^{\prime}, p ; t\right) / \frac{\partial}{\partial q} \int_{0}^{a} \mathrm{~d} q^{\prime} \int_{-b}^{b} \mathrm{~d} p \\
& =(2 b)^{-1} \int_{-b}^{b} \mathrm{~d} p \varrho(q, p ; t),
\end{aligned}
$$



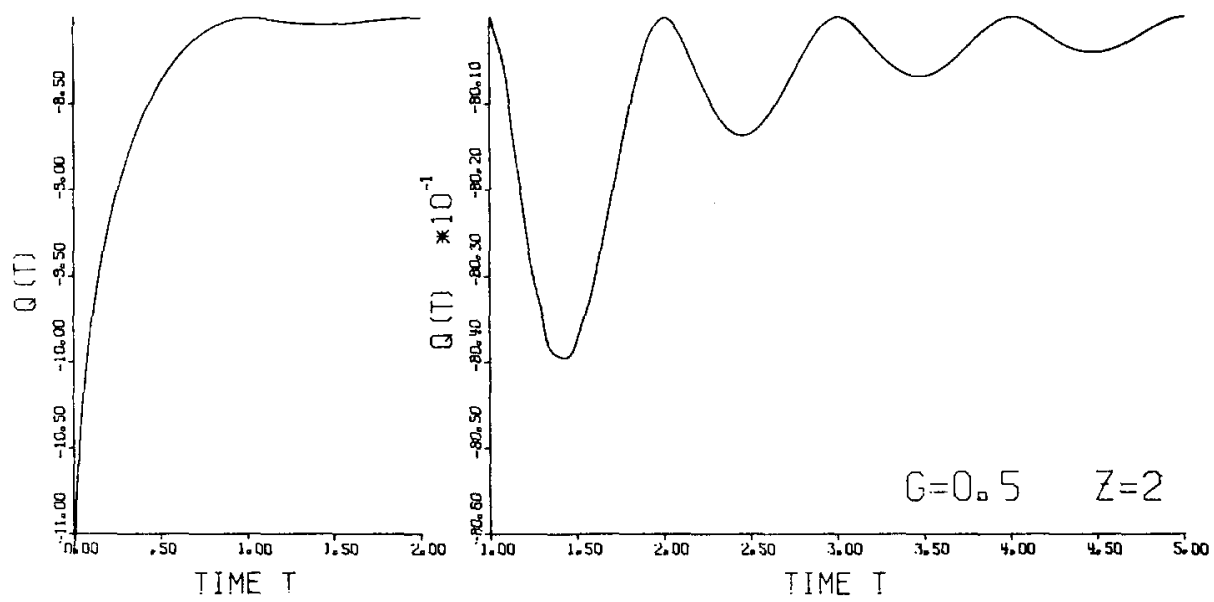

Fig. 4

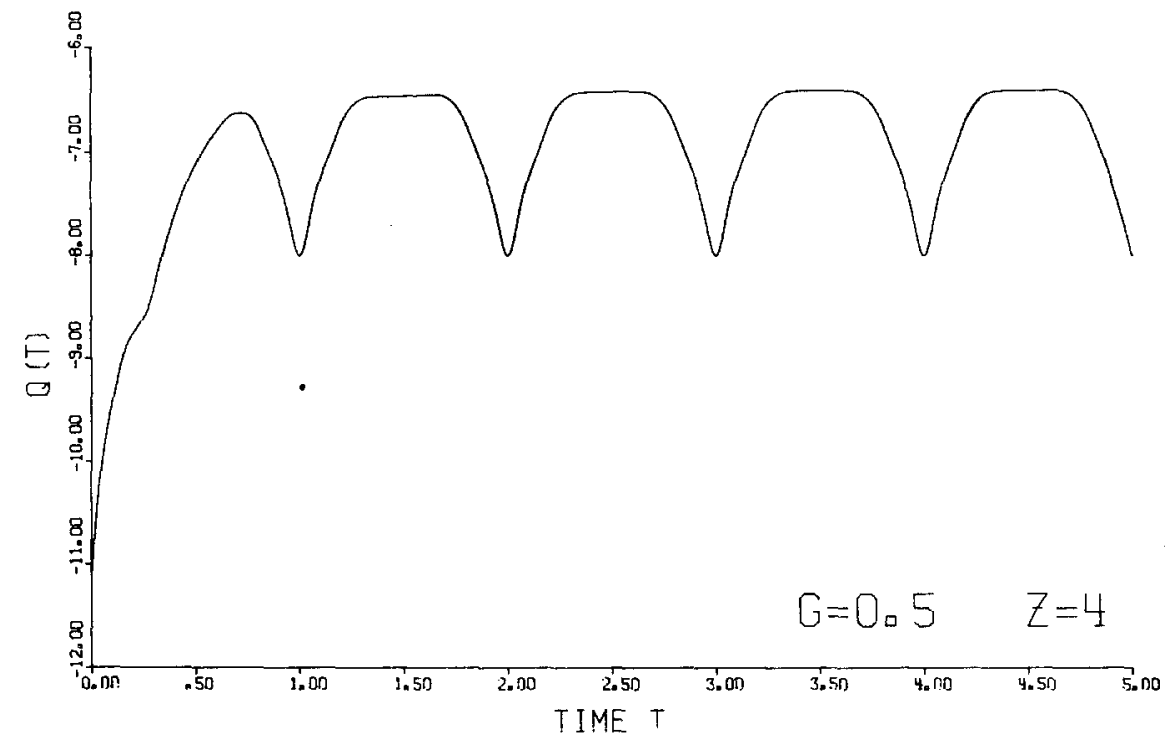

Fig. 5

which is apart from normalization identical with the reduced distribution function. $S_{\mathrm{eg}}^{(\infty)}(t)$ is then calculated according to eq. (4.30); the result for $g=0.5$ is plotted in fig. 1. We note that stability of $S_{\mathrm{cg}}(t)$ against subdivision of the cells is reached fairly quickly; $S_{\mathrm{cg}}(t)$ for $z=8$ or $z=16$ differs from $S_{\mathrm{cg}}^{(\infty)}(t)$ by less than 1.2 or 0.4 percent for $\tau \geqslant 0.5$, or less than 0.05 or 0.02 percent for $\tau \geqslant 1$, respectively. The percentual deviation for very small $\tau$ is larger since $S_{\mathrm{cs}}^{(\infty)}(t)$ is near zero. 
The interchange of the operations $\lim _{t \rightarrow \infty}$ and $\partial^{r} / \partial a_{1} \cdots \partial a_{r}$ in expression (4.32), as discussed in 4.6, is allowed for the model; the reason is that for the free particle integration over $p$ instead of $p$ and $q$ suffices to convert the weak convergence of $\varrho(q, p ; t)$ to strong convergence $\left.{ }^{22}\right)$.

Concluding this section, we note that the model confirms the statements of section 4 about the general case.

\section{Discussion and summary}

Clearly our considerations in this paper could not definitely decide whether the coarse-grained entropy is an appropriate microscopic expression for the entropy for equilibrium and nonequilibrium, the reason being that we did not show that the crucial postulate (P4) is fulfilled. Some indications for the validity of (P4), however, were given. Firstly, it was seen in 4.4 that a modified version of (P4) is in general fulfilled where the empirical relaxation time is replaced by the expectation value of the relaxation time. Secondly we have shown in 4.6 that the common objection that the time behavior of $S_{\mathrm{cg}}(t)$ depends essentially on the choice of the cells is not generally valid. For a fixed set of macroscopic quantities by means of which the system is observed, the variation of $S_{\mathrm{cg}}(t)$ by changing the size of the cells will be the smaller the smaller the cells are. This ensures the existence of an upper bound $\tau_{0}$ on the times after which the $S_{\mathrm{cg}}(t)$ 's reach approximately the equilibrium value.

We could not show that $\tau_{0}$ is of the order of the relaxation time. But this is not unplausible for two reasons: Firstly we have shown in 4.7 that the qualitative dependence of $\tau_{0}$ and of $\tau_{\mathrm{rel}}$ on the number of macroscopic quantities is the same. Secondly, all the arguments that have been put forward in favour of an approach of $S_{\mathrm{cg}}(t)$ to its equilibrium value in due time apply to $S_{\mathrm{cg}}^{(x)}(t)$ as well. The reason being that both some $P(x ; t)$ and $P^{(\infty)}(x ; t)$ are obtained by a coarse-graining procedure that approximates $\varrho(x ; t)$ in an extremely crude way. In the model discussed in section 5 , we have seen that $S_{\mathrm{cg}}(t)$ for 8 or 16 cells does not differ very much from $S_{\mathrm{cg}}^{(\infty)}(t)$ (although one has to be careful with generalizations from this simple model).

\section{Acknowledgements}

I wish to thank A. Thellung in particular, W. Ochs, T. Paszkiewicz and G. Suessmann for fruitful discussions; R. Beck for help in the numerical calculations; and Miss B. Koch for reading the English manuscript. 


\section{References}

1) P. Fong, Physica 30 (1964) 1061.

2) E.T. Jaynes, Am. J. Phys. 33 (1965) 391.

3) C.W.F. McClare, Nature 240 (1972) 88.

4) S. Petrescu, Revue Roum. Sci. Tech. Elektrotech. Energ. (Rumania) 17 (1972) 145.

5) A.J.Hillel, Nature 242 (1973) 456.

6) D. R. Wilkie, Nature 242 (1973) 606.

7) A.C.Legon, Nature 244 (1973) 431.

8) J. Meixner, Physica 59 (1972) 305.

9) I. Prigogine and F.Henin, in: Statistical Mechanics, ed. T.A.Bak (Benjamin, New York, 1967).

10) I. Prigogine, in: The Boltzmann Equation, Acta Phys. Austr. Suppl. X (1973), eds. E. G.D. Cohen and W. Thirring.

11) I. Prigogine, in: The Physicist's Conception of Nature, ed. J.Mehra (Reidel, Dordrecht, 1973).

12) I. Prigogine and F.Mayné, in: Transport Phenomena, eds. G. Kirczenow and J.Marro (Springer, Berlin, 1974).

13) J.Biel, in: Transport Phenomena, eds. G. Kirczenow and J. Marro (Springer, Berlin, 1974).

14) G.Sperber, Found. Phys. 4 (1974) 163.

15) F. Henin, Physica 76 (1974) 201.

16) A.Hobson, Concepts in Statistical Mechanics (Gordon and Breach, New York, 1971).

17) J.U. Keller, Acta Phys. Austr. 35 (1972) 321.

18) J.W. Gibbs, Elementary Principles in Statistical Mechanics (Yale Univ. Press, New Haven, 1902).

19) P.Ehrenfest and T.Ehrenfest, Encykl. Math. Wiss. IV 4 (1911).

20) N.G. van Kampen, Fortschr. Physik 4 (1956) 405.

21) N.G.van Kampen, in: Fundamental Problems in Statistical Mechanics, ed. E.G.D.Cohen (North-Holland, Amsterdam, 1962).

22) H. Grad, Comm. Pure Appl. Math. 14 (1961) 323.

23) R.C.Tolman, The Principles of Statistical Mechanics (Oxford Univ. Press, London, 1938).

24) D.ter Haar, Rev. Mod. Phys. 27 (1955) 289.

25) B.R.A.Nijboer, in: Fundamental Problems in Statistical Mechanics, ed. E.G.D.Cohen (North-Holland, Amsterdam, 1962).

26) G. E. Uhlenbeck, in: The Physicists Conception of Nature, ed. J. Mehra (Reidel, Dordrecht, 1973).

27) R.Becker, Theorie der Wärme (Springer, Berlin, 1955).

28) A. Hobson, Phys. Letters 26A (1968) 649.

29) C. J. Myerscough, J. Stat. Phys. 5 (1972) 35.

30) I. Prigogine, Nonequilibrium Statistical Mechanics (Interscience, New York, 1962).

31) C.T. Lee, Can. J. Phys. 52 (1974) 1139.

32) R. L. Liboff, J. Stat. Phys. 11 (1974) 343.

33) W.Kern, Z. Physik B 20 (1975) 215.

34) V. I. Arnold and A. Aver, Ergodic Problems of Statistical Mechanics (Benjamin, New York, 1968).

35) P.Hoyningen-Huene, Helv. Phys. Acta 46 (1973) 468. 